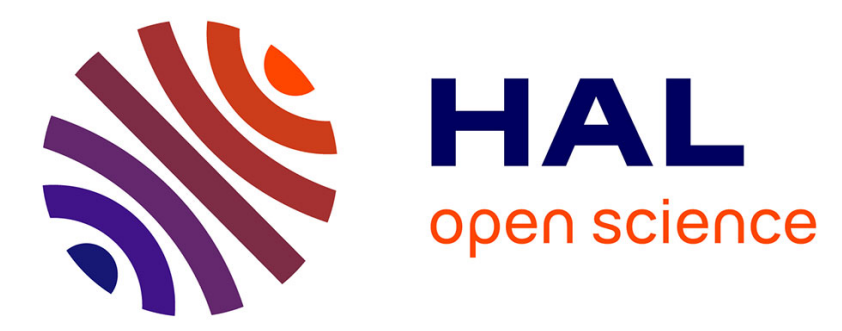

\title{
Action environnementale et démocratie locale en Hongrie post-socialiste \\ Samuel Depraz
}

\section{To cite this version:}

Samuel Depraz. Action environnementale et démocratie locale en Hongrie post-socialiste. Revue d'Etudes Comparatives Est-Ouest, 2005, L'environnement à l'Est. Le modèle européen à l'épreuve, 36 (1), pp.33-61. 10.3406/receo.2005.1695 . halshs-01547057

\section{HAL Id: halshs-01547057 https://shs.hal.science/halshs-01547057}

Submitted on 19 Oct 2017

HAL is a multi-disciplinary open access archive for the deposit and dissemination of scientific research documents, whether they are published or not. The documents may come from teaching and research institutions in France or abroad, or from public or private research centers.
L'archive ouverte pluridisciplinaire HAL, est destinée au dépôt et à la diffusion de documents scientifiques de niveau recherche, publiés ou non, émanant des établissements d'enseignement et de recherche français ou étrangers, des laboratoires publics ou privés. 


\section{Action environnementale et démocratie locale en Hongrie post-socialiste Environmental Policies and Local Democracy in the post-socialist Hungary}

Samuel DEPRAZ

Laboratoire "Mutation des territoires en Europe », UMR 5045 CNRS

\section{Résumé :}

L'histoire des politiques environnementales en Hongrie semble marquée par les caractéristiques du système d'État socialiste : la prise en compte de l'environnement dans la planification est restée, jusqu'en 1989, largement sectorielle et limitée à la seule protection de la nature. Les décisions politiques relevaient du pouvoir central et de la sphère bureaucratique, peu enclins à la régulation des pollutions et des risques environnementaux. Les mouvements écologistes de la décennie quatre-vingts ont précisément contribué à dénoncer ces traits et à ébranler le régime finissant.

Cependant, la période post-socialiste, malgré sa volonté affichée de rupture avec le régime précédent, n'a pas tout à fait gommé les traces des pratiques politiques et institutionnelles antérieures. L'action environnementale a repris un tour technocratique et centralisé ; les institutions politiques peinent à appliquer le discours environnemental européen fondé sur le développement durable et l'initiative locale - précisément parce que cette dernière fait défaut. Le principal héritage du socialisme serait ainsi le manque relatif de participation démocratique à la question environnementale comme à l'action locale en général.

Mots-clés : Hongrie, environnement, post-socialisme, politiques publiques

\section{Summary :}

The history of environmental policies in Hungary seems to be characterized by the principles of the socialist State system : until 1989, the environment was only taken in account as a sectorial policy of the planning process and strictly limited to nature conservation. Political decisions were the matter of the central government and the bureaucratic sphere, which were both few willing to regulate pollutions and environmental hazards. Ecological movements, by the end of the eighties, have precisely contributed to denounce those features and to shake the declining regime.

However the post-socialism has not completely erased the spurs of the previous political and institutional practices, in spite of a strong will to break out with the former regime. Environmental policies have recovered a technocratic-centralizing aspect; official institutions struggle with the European environmental discourse, which is based on sustainable development and local initiative precisely because this last point is missing. Thus the main legacy of the socialist era seems to be the lack of democratical local participation to environmental issues, as to local policies in a more general way.

Key-words: Hungary, environment, post-socialism, public policies. 


\section{Action environnementale et démocratie locale en Hongrie post-socialiste}

Samuel DEPRAZ

Laboratoire «Mutations des territoires en Europe », UMR 5045 CNRS, Montpellier

\section{Introduction}

L'étude des politiques publiques en matière d'environnement, en Hongrie comme ailleurs, prend généralement deux formes : d'une part, l'examen des institutions d'État et des réglementations officielles qu'elles produisent [ČEŘOVSKÝ, (1988) ; FARAGÓ, LAKOS, (1996)], ce qui constitue souvent l'approche la plus simple et la plus officielle de la question; d'autre part, l'étude des mouvements civils ou des acteurs écologistes [PERSÁNYI, (1993) ; SzIRMAI, (1997)] - démarche autrement plus difficile en ce qu'elle implique un travail de terrain pour évaluer les positionnements idéologiques des personnes et des groupes sociaux. Pourtant, il semble que l'histoire de l'environnement se tisse au croisement de ces deux écheveaux : si l'activité législative et institutionnelle d'un État peut traduire les tensions sociales qui s'y expriment, à l'inverse l'action militante révèle la nature de l'action publique et ses manques éventuels.

En Hongrie, l'histoire de l'environnement peut se décrire par un balancement d'ensemble d'un pôle vers l'autre : les politiques centralisées et bureaucratiques du système d'Etat, encore sectorielles et limitées, cèdent leur place avec la rupture de 1989 à l'expression démocratique des organisations sociales en environnement et à une action plus globale et participative.

Pourtant, ce mouvement idéal est quelque peu forcé par la lecture trop appuyée des discontinuités politiques : les temporalités du changement social sont plus fines et les ruptures ne sont bien souvent qu'apparentes. Ainsi, l'examen détaillé de l'action environnementale du socialisme traduit une évolution plus graduelle vers l'affirmation des revendications civiles. Le dialogue s'est amorcé dès les années soixante et - sans que l'État ne se départisse totalement de son caractère autoritaire - culmine malgré tout avant 1989. Les politiques environnementales participatives du post-socialisme ont été préparées, semble-t-il, avant la rupture systémique. De même, rien ne garantit que l'action environnementale ait totalement rompu aujourd'hui avec l'héritage du système d'État socialiste.

Tout en respectant la lecture conventionnelle allant du socialisme au post-socialisme, il semble donc utile de prêter attention aux logiques de continuité et d'héritages dans ce dialogue entre secteur institutionnel et organisations civiles en environnement. 


\section{Le fonctionnement de l'action environnementale hongroise sous le socialisme}

L'histoire de l'action environnementale du régime socialiste en Hongrie est marquée par le mode de fonctionnement même du système d'État. C'est-à-dire que l'environnement a été envisagé comme un principe d'action sectoriel parmi d'autres tandis que la planification prônait par ailleurs l'intensification et le développement économique du pays. Il n'y a pas eu prise en compte globale des interactions homme-société mais au contraire développement de deux logiques simultanées et découplées dans l'espace : protection de la nature d'une part, intensification sans mesure de la production d'autre part.

L'absence de toute approche systémique respectueuse des spécificités locales et d'éventuelles revendications sociales nous semble intrinsèquement liée à la nature initiale du régime : centralisé, autoritaire et bureaucratique. Ces trois caractéristiques transparaissent dans l'analyse historique de l'action environnementale socialiste, que ce soit dans la gestion des pollutions, dans le développement de la protection de la nature ou dans la période tardive d'affaiblissement de l'État et d'émergence d'un débat démocratique en Hongrie.

\subsection{Des pollutions révélatrices du fonctionnement du système d'État}

Le bilans écologiques dressés immédiatement après la chute du système d'État permettent de dresser aujourd'hui une géographie relativement précise du legs socialiste en Hongrie. Tout d'abord, les orientations de la planification socialiste en faveur de l'industrie lourde et de l'utilisation domestique du charbon ont créé, en Hongrie comme ailleurs en Europe centrale, des zones de concentration des déchets industriels et des polluants atmosphériques ou terrestres autour des grandes métropoles et des complexes sidérurgiques intégrés [SÁNDOR, 1993 ; FoDOR, 1991]. Cet héritage du socialisme est sans doute le plus connu, même si les excès marquants qui en ont découlé ont disparu du fait du déclin voire de la fermeture de certaines industries et de la modernisation des équipements domestiques. Cependant certains polluants, comme les métaux lourds, demeurent dans les sols et seront longs à éliminer.

Fait moins souvent étudié, le milieu rural agricole n'est pas plus épargné : durant le socialisme, dans une volonté de modernisation et d'intensification de l'agriculture, l'utilisation d'engrais s'est généralisée à partir de la fin des années soixante dans toutes les exploitations collectives de la seconde collectivisation. Mais le faible coût des intrants chimiques a suscité de nombreux gaspillages, si bien que $75 \%$ des nappes phréatiques de 
surface de la Grande Plaine hongroise (Alföld) sont devenues impropres à la consommation [FODOR, id.] tandis que leur niveau moyen s'est abaissé localement de $20 \mathrm{~m}$ en raison des prélèvements excessifs pour l'irrigation [KERTÉSZ, 1999] ; or la plupart des ménages ruraux n'avaient pas accès à un réseau d'adduction d'eau potable en 1990 et puisaient directement dans ces nappes. Enfin, la mécanisation de la production agricole, trop agressive pour les sols les plus sableux, a entraîné une déflation éolienne significative [KERTÉSZ, 1981 ; SzABó et al., 1994]. Enfin, la politique d'afforestation de l'Alföld entamée au début du siècle pour fixer les sables, enrichir les sols et favoriser le développement des cultures a été poursuivie sans que soit réellement discutée la question de la capacité du milieu à supporter ces changements exogènes. C'est-à-dire que la spécificité des écosystèmes steppiques, milieu naturel fragile propice à une mise en valeur extensive, n'a pas résisté aux politiques d'intensification socialistes. Les secteurs les plus épargnés sont encore les reliefs collinéens du sud-ouest du pays et les espaces marécageux résiduels de la Grande Plaine [carte].

Le cœur du problème écologique réside dans la structure même du pouvoir socialiste. Dans un premier temps, il n'y a eu aucune prise en compte des capacités d'auto-épuration et de renouvellement de la ressource naturelle. L'objectif productiviste affiché de la première planification quinquennale 1950-54, ajouté au faible intérêt du pouvoir d'obédience stalinienne dans la recherche en environnement, peut expliquer cela : la période était très défavorable à la pensée environnementale [BATOU, 1992]. En effet, le paradigme dominant de cette époque restait strictement fidèle aux textes marxistes de base qui postulaient la «maitrise de la nature par l'homme » comme préalable à la révolution communiste - ce que l'on peut qualifier de «nihilisme environnemental »[ FleTSCHER, POMAZY, in VINAVER, 1993]. La nature était donc envisagée comme une ressource gratuite, support du développement humain.

Avec la déstalinisation, le discours des scientifiques sur l'environnement a pu se faire entendre à nouveau mais le système socialiste s'est avéré malgré tout incapable de corriger les déprédations environnementales: les premiers textes de loi sanctionnant les entreprises polluantes ont été généralement contournés ; les sanctions n'étaient "payées qu'en paroles » [SÁNDOR, (28), 1993]. Ceci réside dans la logique du système d'État, perverti à la base puisque "l'organe régulateur et contrôleur en matière d'environnement était l'État, c'est-àdire celui-là même qui possédait et développait les outils de production économique» [DEPRAZ, KERTÉSZ, (420), 2002]. Enfin, la société civile, fortement encadrée par le pouvoir central, n'avait qu'une faible capacité de réaction face à la dégradation de son milieu de vie - 
tout au moins jusqu'aux années quatre-vingts. Lorsqu'il en était question, elle se heurtait généralement à l'incurie bureaucratique.

\subsection{Protection de la nature sans protection de l'environnement}

En revanche, la protection de la nature, entendue comme la protection des seuls écosystèmes naturels ou des espèces menacées, a été un réel axe politique sous le socialisme. C'est-à-dire qu'il y a bien eu protection de la nature, sur certaines portions du territoire national, mais sans protection effective et globale de l'environnement.

L'émergence d'une politique de protection de la nature en Hongrie est liée à la politique de réconciliation civile kádáriste après le traumatisme de 1956. Amnistie de 1963, déstalinisation partielle du pouvoir, introduction de la seconde économie en 1968, la période a été propice aux concessions. Le paradigme politique dominant du pouvoir a changé : le développement hongrois passe désormais par une plus grande participation citoyenne et par l'acceptation d'une critique constructive du régime. C'est dans cet élargissement de la sphère civique que se développent les premiers mouvements environnementaux et qu'une évolution législative est possible.

Premier jalon, un Office national de protection de la nature (Országos Természetvédelmi Hivatal) est instauré en 1962, structure gouvernementale dépendant directement du pouvoir central. La loi sur les parcs nationaux est promulguée en 1971 et suivie de la création des deux parcs de l'Hortobágy en 1972 et du Kiskunság en 1975. Dans le même temps, la société ornithologique hongroise (Magyar Madartány és természetvédelmi Egyesület), fondée en 1974 et associée au mouvement international Birdlife, devient le groupe le plus influent en Hongrie puisqu'elle compte 15000 membres autour de 1980. Tournée vers l'action environnementale, elle suscite de nombreux groupes locaux et agit réellement comme une fédération nationale. Les scientifiques de l'Académie des Sciences agissent également en faveur de l'environnement au travers des plans quinquennaux. L'Académie lance, durant la décennie soixante-dix, un plan d'assainissement du lac Balaton resté célèbre et mis en œuvre à partir de 1983 ; elle organise aussi une conférence nationale sur l'environnement. C'est également l'Académie qui a déterminé les emprises des zones protégées des parcs nationaux, tandis que la société ornithologique hongroise s'est impliquée dans la gestion du parc du Kiskunság. Il existe donc à partir de cette période une réelle volonté politique en matière de protection de la nature impliquant un minimum de participation civile. 
Pourquoi un tel écart ? Contrairement à la réparation des pollutions ou à une prise en compte globale de l'environnement dans le développement économique, la création d'espaces protégés est une concession facile à la demande sociale. Les premiers territoires protégés ont été ceux qui formaient le cœur des représentations hongroises sur la puszta et auxquels les hongrois pouvaient être les plus attachés, en témoignent les représentations artistiques des steppes de la Grande Plaine au dix-neuvième siècle. La symbolique patrimoniale et identitaire du statut de «parc national » pouvait répondre adéquatement à cette attente. Du point de vue scientifique, ce sont également ces terrains qui ont été investis de longue date par les chercheurs budapestois et par les universitaires de Szeged ou de Debrecen : on recense des études en biologie sur l'Hortobágy et le Kiskunság dès 1800, avec un pic entre 1870 et 1910 . Le classement d'un secteur du Kiskunság en réserve naturelle protégée a même été proposé dès 1934 [SZUJKÓ-LACZA, 1993]. L’attente scientifique a donc elle aussi été comblée.

Cependant, le tracé des parcs nationaux sur le terrain peut étonner : il correspond à une logique d'échantillonnage des espaces naturels, si bien que les parcs sont éclatés en de multiples petites unités destinés à l'étude scientifique, comme autant de laboratoires en plein air. Cette mosaïque semble contraire à toute logique élémentaire: si l'on multiplie les coupures entre unités, l'interface entre les zones protégées et l'extérieur s'en trouve d'autant rallongée, l'influence anthropique s'y étend. La réflexion écologique prône également de nos jours le maintien de corridors écologiques, ou stepping zones, entre plusieurs sites naturels afin de préserver la mobilité des espèces entre différents habitats. Mais cette dimension est plus contraignante en ce qu'elle impose une continuité dans les territoires protégés. Elle n'a ainsi pas été retenue lors de la création des parcs hongrois.

En effet, le choix de créer des "parcs-patchworks» [MOLNÁR, 1998] s'avère très avantageux pour l'État: l'éclatement permet de préserver toutes les parcelles de terrain propices à l'agriculture dans la mesure où les espaces concernés par les parcs présentent tout de même une occupation humaine non négligeable. "En Hongrie, en raison des conditions économiques et sociales antérieures, la protection de l'environnement se fondait sur la protection des ressource inexploitables et non sur une approche écologique ou sanitaire » [MezöSI, Mucsi (99), 1993]. Ainsi, l'ensemble étonnant que constitue le parc national du Kiskunság, éclaté en neuf unités de gestion parfois distantes de plusieurs dizaines de kilomètres, s'explique assez simplement par l'intercalation d'exploitations agricoles intensives alors sous contrôle de coopératives et de fermes d'État. Ces vastes parcelles étaient précisément situées sur des pastilles fertiles de loess réparties en mosaïque sur ce secteur de la 
Grande Plaine. N'ont été placés sous protection que les marécages interstitiels, les bassins sodiques et les sables éolisés - autant de terrains inutiles du point de vue de la planification économique. Il en va de même dans l'Hortobágy, où le parc, quoique moins dispersé, compte tout de même 21 unités différentes.

Parcs naturels à vocation scientifique, les parcs nationaux hongrois n'ont de ce fait pas bénéficié d'une mise en valeur touristique ou sociale durant le socialisme : peu de tourisme de nature, peu d'infrastructures d'accueil ou de signalétique. Seule l'éducation à la nature des enfants des écoles riveraines des parcs y a été développée. En revanche, la production scientifique attachée aux parcs a été et demeure relativement abondante [SZABó, 1985; TóTH, 1995]. En somme, la protection de la nature en Hongrie a su trouver une expression politique sous le socialisme, quoique partielle et concédée à une demande sociale grandissante dans la mesure où elle ne devait pas contrevenir aux priorités de la planification d'État.

\subsection{L'utilisation politique croissante de l'action environnementale}

Autre caractéristique du socialisme, la protection de l'environnement a revêtu au fil du temps un rôle politique accru sur la scène intérieure ou à l'international. En créant des parcs nationaux et une législation sur l'environnement, que cela soit efficient ou non sur le terrain, la Hongrie s'est distinguée comme un État pionnier lors de la première conférence mondiale sur l'environnement à Stockholm, en 1972. L'État socialiste montre qu'il prend l'environnement au sérieux en s'insérant dans les réseaux internationaux de protection de la nature : le programme Man and Biosphère de l'UNESCO est adopté en 1970, la convention Ramsar sur les zones humides est ratifiée en 1971 et le premier terrain d'application de ces deux traités est le lac Fertő - ou Neusiedler See - terrain symbolique puisqu'il est frontalier avec l'Autriche. La loi sur l'environnement humain de 1976, actualisation de celle de 1961, met également la Hongrie à l'unisson avec les autres pays européens dans cette période de genèse internationale d'un droit de l'environnement.

Pour affronter à la fois la critique intérieure et montrer l'exemple face au bloc capitaliste, l'État hongrois met en œuvre à partir de cette même période une politique du chiffre, avec des objectifs quantifiés dans le domaine de la protection de la nature. En 1976 est lancé, conformément à la logique planificatrice du système, un programme spécifique à l'environnement avec des objectifs chiffrés sur 15 années. De même, les bilans et histoires de l'environnement mettent en avant à partir de cette époque l'importance de la surface nationale couverte par un espace protégé : avec 4\% de son territoire protégé dès 1976 et $7 \%$ en 1990, la 
Hongrie devient en effet le leader européen pour la superficie de ses espaces naturels protégés. Dans une même volonté démonstrative, l'Atlas national de 1988 fait paraître une courbe retraçant la croissance exemplaire des parcs et réserves en Hongrie et même les objectifs prévus d'ici la fin du plan [document 1].

Cette course au chiffre est toutefois à double tranchant : les organisations écologiques hongroises peuvent également s'en saisir pour montrer les insuffisances ou le ralentissement des politiques publiques en matière de protection de la nature.

C'est ainsi que l'on peut éclairer la «fièvre législative» qui apparaît en matière d'environnement lors de la décennie 1980: l'État socialiste, affaibli par ses difficultés économiques et sa dette démesurée, se prend dans l'engrenage des revendications écologistes et doit surenchérir dans la création de réserves naturelles, dans l'adoption de textes de loi ou dans la ratification de conventions internationales. Réforme de l'Office national et du Conseil national de protection de la nature en 1977, création en 1980 d'une agence de mesure et d'étude en environnement (KVI) employant près de 200 personnes, nouveau décret sur la protection de la faune et de la flore en 1982 [ČEŘOVSKÝ, 1988], signature de 18 traités internationaux entre 1979 et 1989: la politique environnementale de l'État s'accélère [document 2] - tout en donnant de la sorte une importance toujours croissante aux contestataires.

Ceci peut, en effet, expliquer la prise d'autonomie grandissante des mouvements environnementaux à partir de 1980. Leur contestation rencontre un écho de plus en plus vif auprès des populations civiles par effet «boule de neige », de victoire locale en victoire locale [document 3]. Ce n'est pas tant l'argument écologique qui prime que le besoin d'expression démocratique locale. Les groupes écologistes constituent alors en effet une des rares brèches où est possible une critique radicale du système socialiste chancelant, sous couvert d'intérêt général et de protection de l'environnement : il s'en crée ainsi deux par mois en 1988.

Quels sont donc ces groupes qui ont contribué à faire vaciller le régime socialiste hongrois durant la décennie quatre-vingts ? L'activité écologiste hongroise a pris son essor au sein de trois milieux sociaux voisins et intersectés : les étudiants, les urbains et les cercles intellectuels ou scientifiques.

Le ferment étudiant se trouve sans doute au sein de l'université des Sciences Eötvös LORÁNT (ELTE) de Budapest. En 1983, de jeunes biologistes fondent le groupe de protection 




document 1 : l'évolution des superficies protégées en Hongrie (en \% de la superficie nationale et en ha) Source : PÉCSI M. (dir.) (1989), Magyarország Nemzeti Atlasza, Académie des Sciences, Budapest, 132 p.

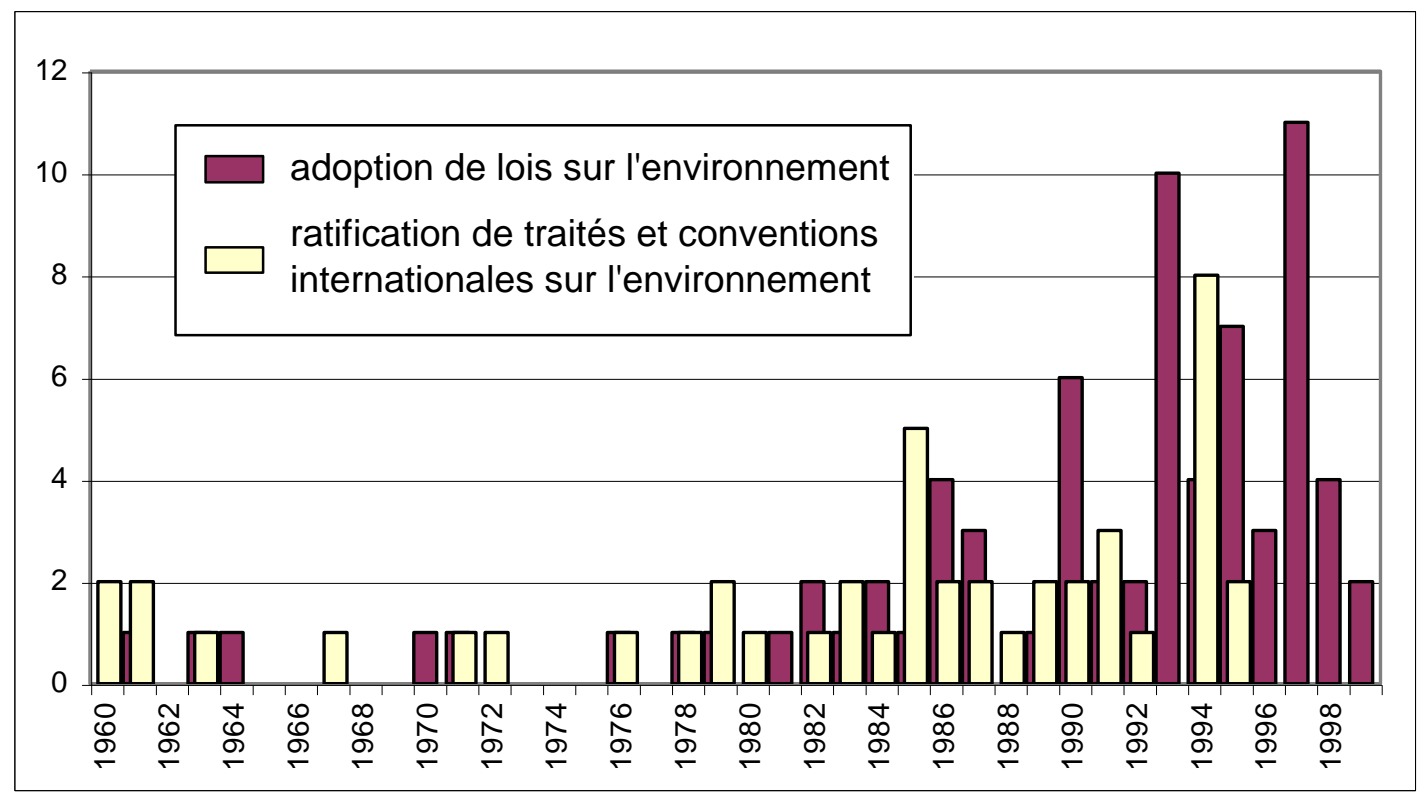

document 2 : l'évolution de l'activité législative de la Hongrie sur la scène nationale et internationale, périodes 1960-1999 (lois) et 1960-1995 (conventions) - Sources : MARTHA (1999) ; FARAGO, LAKOS (1996) 
de la nature (ELTE Természetvédelmi Klub) en réaction à un projet de mine d'extraction calcaire dans les collines méridionales de Szársomlyó.

Cette organisation prône la désobéissance civile, mène des campagnes de sensibilisation dans les écoles et édite des documents d'information [PERSÁNYI, 1990]. De même, le «Cercle vert » de l'université technique de Budapest (BME Zöld Kör) s'attaque à la pollution atmosphérique de la capitale. La faculté de Médecine s'organise également, tandis que d'autres groupes écologistes se développent simultanément dans les établissements universitaires de province : université agronomique de Gödöllö, université forestière de Sopron, facultés de biologie de Debrecen et de Szeged. A partir de 1986, les groupes étudiants sont suffisamment organisés pour fonder un réseau européen, Greenway, sur l'initiative de l'ELTE Klub.

Les groupes urbains sont, eux, plus radicaux et apparaissent surtout après 1985 : REFLEX (Győr), E-MISSZIO (Nyíregyháza), Göncöl Alapítvány (Vác), Holocén (Miskolc), Mosonmagyaróvári Környetvédők (Mosonmagyaróvár) [REC, 1997]. Le plus important fut toutefois le Mouvement du Danube, groupe fondé en 1984 et fédérant diverses organisations de l'agglomération budapestoise (Duna Kör, Dunaért Alapítvány, Kékek). Mêlant civils, scientifiques et organes de presse, il s'est heurté directement au gouvernement central en dénonçant le gigantesque projet hydroélectrique international de Gabčikovo-Nagymáros, sur le Danube. Des représailles sur ses membres (intimidations, pertes d'emploi) ne l'ont pas empêché de gagner une pleine visibilité et un soutien financier international : le Mouvement du Danube gagne le Right Livelyhood Award en 1985, prix Nobel alternatif, et le Goldman Prize en 1990 [SZIRMAI, 1997]. Ce fut la seule organisation environnementale à rencontrer un large soutien populaire avec plus de 10000 membres en 1988. Autant de signes d'un renforcement de la contestation civile et des fissures du système d'État.

Du côté scientifique, l'action fut moins directe. Le cercle scientifique interdisciplinaire étudiant (Interdiszciplináris Tudományos Diákkör), créé en 1981, a organisé des conférences et des ateliers sur l'environnement. Il s'agissait surtout d'un mouvement d'idées alternatif voire religieux, s'inspirant du mouvement idéaliste Bokor, fondé au sortir de la guerre. Les scientifiques de l'Académie des Sciences, quoique tenus à une certaine réserve, ont eux aussi animé un groupe de vulgarisation des connaissances auprès d'un public national ciblé. Ils ont tenu un comité permanent sur l'environnement, ont publié des rapports et ont été dépêchés 
comme arbitres dans le conflit du Danube. Leur rôle d'expertise et de médiation a ainsi été reconnu lors de cette période [PERSÁNYI, 1990].

Le parti socialiste ouvrier hongrois a tôt tenté de récupérer l'ensemble de ces mouvements en instillant dans le Front patriotique du peuple (Hazafias Népfront), organisation sociale de masse fondé en 1954, des valeurs écologistes. En est issu également le «Mouvement pour l'environnement des étudiants des universités et grandes écoles ». Ces deux organes du parti ont tenté de limiter l'action environnementale à une sensibilisation du public aux enjeux écologiques.

Plus efficace, le comité central de l'organisation des jeunesses communistes, le KISZ, a créé au début des années quatre-vingts le «Conseil de la jeunesse sur la protection de l'environnement» (Ifjúsági Környezetvédelmi Tanács). Ce fut à la fois une aide et une gêne pour les activistes car la structure centralisée de ce groupe a été très néfaste à la construction d'une opposition politique écologiste. Alors que la contestation s'est accélérée dans la seconde moitié de la décennie quatre-vingts, l'IKT réussit en 1987 à regrouper les mouvements écologistes en une fédération sous son contrôle, la «Société hongroise des protecteurs de la nature »(Magyar Természetvédök Szövetsége), empêchant ainsi toute évolution en direction de la sphère politique.

Si bien que les dissidents n'ont lancé les premiers partis écologistes qu'après 1990, qui plus est en se dispersant en de multiples groupes : Magyar Zöld Párt (1990), Zöld Szocialisták (1993) puis Zöld Alternatíva (1995), aujourd'hui Zöld Demokraták, Magyar Szociális Zöld Párt, Környezetvédök Szociáldemokrata Pártja ou la Zöldek Szövetsége pour les plus importants partis. Ces divergences de fond, apparues au moment précis où l'État socialiste s'effondrait et jamais résolues depuis lors, ont hypothéqué toutes les chances des mouvements écologistes de participer à la nouvelle donne politique et démocratique du pays.

\section{Les temps du post-socialisme, entre rupture et rémanences}

La décennie quatre-vingts ainsi que la rupture systémique de 1989 font ressortir en miroir les caractéristiques de l'action environnementale sous le socialisme : une politique sectorielle et centralisée, limitée à la fois par les priorités économiques du régime et par le manque d'expression démocratique à l'échelon local. Partant, c'est l'idée de rupture qui semble caractériser les politiques environnementales du nouveau système. 


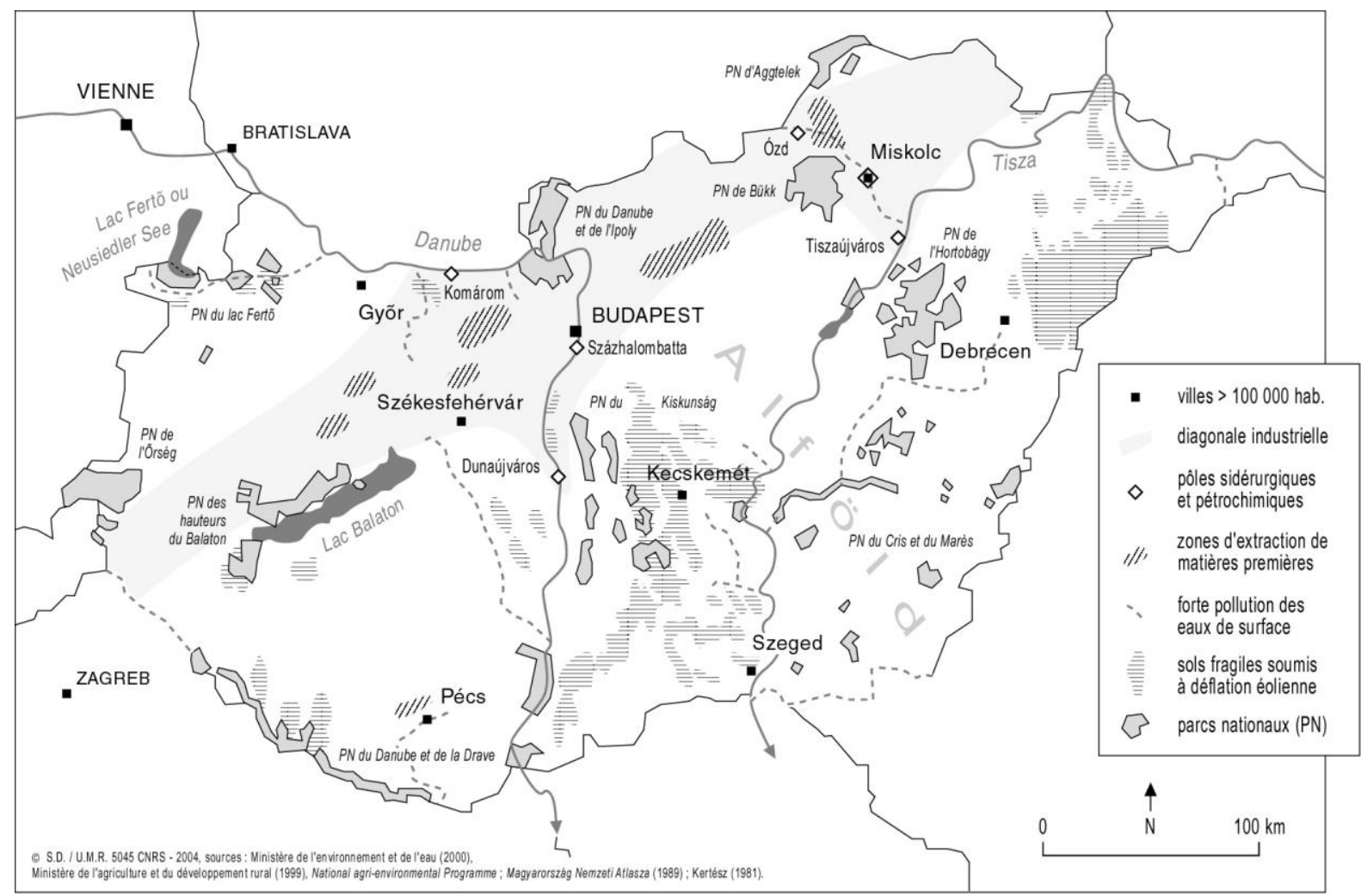

document 3 : carte de la Hongrie.

\subsection{Une volonté de rénovation du discours environnemental}

L'enjeu essentiel du post-socialisme, en matière de protection de la nature et d'environnement, semble avoir été avant tout d'afficher la différence envers le précédent régime. On appuie avec insistance sur le passif légué par le régime communiste, ne serait-ce que pour placer les nouvelles actions publiques sous le signe de la reconstruction et de l'amélioration. La municipalité de Budapest fait ainsi apposer à l'entrée des lignes de métro et aux principaux carrefours de la capitale des panneaux d'information sur la qualité de l'air [SÁNDOR, 1993]. Signal simple et évident, puisque l'installation de ces mêmes panneaux avait été refusée par le régime précédent en 1989.

La Hongrie s'est également dotée de nouveaux symboles historiques. Ainsi, le Ministère de l'éducation a créé un prix Károly KAÁN en faveur de la sensibilisation des enfants à l'environnement, du nom d'un célèbre biologiste et forestier hongrois (1867-1940), secrétaire d'État au début du siècle, auquel on doit à la fois l'afforestation de la Grande Plaine hongroise et la première loi sur la protection de la nature, en 1935. De même, l'historiographie hongroise sur l'environnement remonte aux prémices historiques, depuis les règlements 
royaux de Sigismond sur la gestion des forêts (1426) jusqu'aux décrets de Charles III sur la chasse (1729) et place le cœur de l'élaboration des politiques environnementales nationales entre le compromis austro-hongrois de 1867 et la deuxième guerre mondiale [TARDY, 1996]. La simultanéité de la pensée écologiste hongroise avec l'Europe occidentale est ainsi rappelée ; pourtant, ces textes de loi demeurent d'une portée limitée puisqu'ils se cantonnent à la protection des forêts, des «monuments naturels ${ }^{1}$ et de certaines espèces parmi les plus emblématiques selon une approche de l'environnement encore fortement imprégnée de pittoresque et d'esthétique romantique. La période socialiste, quant à elle, est surtout dépeinte sous son travers bureaucratique, avec une gestion strictement planifiée et institutionnelle de la nature [PAJER, 1992]. Elle fut tout de même porteuse des premières études réellement écosystémiques.

Certes, cette volonté de rénovation de l'histoire environnementale nationale fut surtout sensible durant les premiers temps de la transformation systémique. Elle a toutefois servi de table rase idéologique et de catalyseur à l'établissement de nouvelles figures sur la scène politique et sociale en matière d'environnement.

En effet, dans la droite ligne des mouvements citoyens de 1989-1990, les instances gouvernementales ont favorisé la publication de nombreux bilans écologiques du socialisme, que ce soit sous la direction du Ministère de l'environnement, de l'Académie des Sciences de Hongrie ou de partenaires institutionnels étrangers, notamment le programme des Nations Unies pour l'environnement (UNEP) et la protection de la nature (UICN). On peut ainsi compter, sur le modèle du rapport KASSENBERG/ROLEWICZ de 1985 en Pologne [GUERMOND, Mathied, 1991], une dizaine d'états des lieux représentatifs du regain d'intérêt officiel en faveur de l'environnement en Hongrie. Le besoin de liberté d'expression était, dans ce domaine, similaire à celui des autres sciences sociales [KNABE, 1989] tandis que la tonalité critique des rapports est alors à l'unisson avec le sentiment national et flatte le besoin de changement - et de financements étrangers [ENYEDI, HINRICHSEN, 1990 ; BOCHNIARZ, 1992 ; FODOR, WALKER, 1994 ; RAKICS, KOVÁCS, 1996 ; MiNISTERE, 1996].

La critique la plus poussée vient sans doute des chercheurs en biologie, des géomorphologues et des biogéographes : autant de chercheurs qui, par leur manifeste, ont du même coup réussi à se placer avantageusement sur le marché de la recherche internationale. Autrefois cantonnés à l'examen strictement physique des milieux et des paysages, dans la lignée des sommes géographiques de Márton PÉCSI, ils sont aujourd’hui incontournables dans

\footnotetext{
${ }^{1}$ Selon les termes mêmes de la loi de 1935 (1935. évi IV. törvény az erdőkröl és a termeszetvédelemröl, loi sur les forêts et sur la protection de la nature).
} 
les programmes internationaux en environnement et société, preuve que le signal politique qui a suscité ces rapports répondait aussi à une programmatique personnelle évidente.

La rupture semble donc surtout un paradigme ambiant qui vaut surtout pour le repositionnement de chaque acteur sur la scène politique et scientifique de l'environnement. Reste à voir si les convictions et les actions répondent vraiment à cette idée de rupture.

\subsection{Une désaffection marquée de la société hongroise envers l'environnement}

Car, à l'inverse du choix appuyé du politique en faveur de l'environnement, la conscience environnementale de la population hongroise est notablement retombée. À dire vrai, la récession transformationnelle de 1993-1996 et, en milieu rural, l'effondrement de moitié de la production agricole puis le chômage de la main d'œuvre des entreprises décollectivisées jusqu'à 30\% en 1995-96 en milieu rural - ont fait dire que «le moment n'était pas à l'environnement» [CSATÁRI, 1995]. Ces années difficiles ont entraîné des constats désabusés: "La société n'est pas sensible à l'environnement et le degré de conscience environnementale est bas. Les intentions sont assez limitées et l'observation des décrets existants, c'est caractéristique, n'est pas générale. Les estimations des impacts environnementaux font rarement partie des programmes d'aménagement » [MEZÖSI, MuCSI (99),1993]. Si une majorité de hongrois reconnaît volontiers l'importance de l'environnement, $71 \%$ affirment ne pas être prêts à s'engager en faveur d'une cause écologiste [ORÓsZ in FODOR, (1993)]. Même désaveu, les nouvelles entreprises hongroises préfèrent intégrer dans leurs coûts de production le paiement des amendes plutôt que la prévention des pollutions issues de leur activité de production [SÁNDOR, 1993].

En outre, l'élan apparent des années 1980 en faveur de l'environnement était aussi largement conjoncturel. Porté par le besoin d'expression démocratique local, il s'est vite essoufflé dès lors que la démocratie est devenue la norme de la vie civique. Des 10000 personnes engagées dans le mouvement du Danube, il n'est plus rien resté deux ans plus tard. L'organisation n'a pas su se renouveler après l'abandon du projet hydroélectrique fin 1988. La conférence "Greenwave », destinée à en élargir les centres d'intérêt, n'a pas réussi à fédérer les adhérents autour d'un nouveau projet précis [LÁNG-PICKVANCE, 1997]. Dans le même temps, tous les nouveaux partis hongrois ont senti la nécessité d'intégrer à leur discours un volet environnemental, en particulier le Forum démocratique (centre-droit), le Fidész (droite) et le parti libéral. L'écologie rentre ainsi dans le rang du discours politique - sans que soit assurée pour autant la pérennité de cette conversion. 
Il en a été de même ailleurs en Europe : la mode était partout à l'écologie politique militante au tournant de 1990, en témoignent les scores électoraux élevés des partis écologistes aux scrutins de cette période. Cette mode est largement retombée depuis : l'action environnementale a été institutionnalisée et reprise dans les procédures européennes, qu'il s'agisse des fonds structurels ou des mesures agri-environnementales de la PAC. La technocratisation de l'environnement n'ayant rien d'exaltant, la pression citoyenne s'en est ressentie, en Hongrie comme ailleurs.

Enfin, une certaine satisfaction matérialiste à entrer dans l'économie de marché empêche également la société hongroise d'en critiquer trop vite les excès environnementaux : «les mouvements écologistes ne sont pas suffisamment puissants et organisés pour imposer une tabula rasa dans les théories qui régissent les politiques de développement: la société de consommation fut si enviée pendant des décennies qu'il est impossible de 'brûler cette étape’ » [KAMPIS, 2003]. Les motifs de désaffection sont donc pluriels et cumulés.

Conséquence immédiate au plan politique, les partis écologistes déclarés n’ont jamais obtenu le moindre mandat électif. En 2002, seuls 7 candidats écologistes se sont présentés aux élections législatives; le meilleur score fut obtenu à Pest avec 1,9\% des voix. A l'échelon local, un seul maire sur 3600 environ affiche aujourd'hui une étiquette écologiste dans un contexte marqué, il est vrai, par la dépolitisation croissante des élus locaux. La population civile a pu également être sensible aux dissensions au sein des groupes écologistes, toujours divisés lors des élections parlementaires. L'apparition d'un groupe fasciste au sein de la mouvance écologiste du Magyar Zöld Párt eut un effet tout autant désastreux [FoDOR, WALKER, 1994]. Certes, plus d'une centaine de membres du Cercle du Danube ont obtenu un mandat en 1990: mais il s'agissait avant tout de personnalités politiques locales ou médiatiques et toutes ont été élus sous une étiquette politique non-écologiste.

Pour prolonger leur action dans ce contexte devenu défavorable, les responsables écologistes les plus charismatiques des mouvements de 1989 ont emprunté alors deux trajectoires divergentes : soit celle de leur intégration aux réseaux dominants du pouvoir, soit celle du basculement dans la sphère associative.

Ceux qui se sont intégrés aux grands partis politiques ont, du même coup, perdu leur liberté d'action offensive en s'engageant dans des structures institutionnelles parfois éloignées de la scène hongroise, notamment européennes (cf. György DrOPPA, leader du Cercle du Danube depuis 1990). Leur succès politique au plan international traduit l'intérêt que l'on 
porte à l'action environnementale hongroise, notamment dans les ONG anglo-saxonnes et les instances européennes - intérêt largement supérieur à celui suscité en Hongrie même.

Sur la scène nationale, l'action écologiste militante est donc plutôt passée dans la sphère associative, avec un net repli vers l'échelon local. Ainsi l'une des anciennes militantes du cercle du Danube a quitté la sphère politique pour fonder le Centre indépendant sur l'écologie (Független Ökológiai Központ). Cette fondation budapestoise, l'une des plus importantes ONG environnementales du pays, met en œuvre des projets de développement durable à l'échelon des micro-régions hongroises et organise des séminaires de formation pour les acteurs locaux avec l'aide d'un financement suisse et de la fondation Soros. Le WWF et les Verts européens continuent à coopérer avec le Cercle du Danube, devenu un groupe d'expertise sur les questions relatives à l'eau. Enfin, la société ornithologique, devenue Ligue de protection de la Nature-Birdlife Hungary en 1989, conserve son influence nationale avec des antennes à Borsod, Győr, Csongrad et Szabolcs, tout comme la Société hongroise des protecteurs de la nature, forte de 6000 membres et de 63 antennes nationales.

Ceci étant, les associations de défense de l'environnement se sont certes multipliées depuis 1989 - il s'en est créé une trentaine par an depuis lors - mais 66\% de ces organisations civiles sont implantées dans des petites villes et comptent moins de 25 membres; seules 6 à 8 dépassent la centaine de membres. Leur financement est dérisoire : 75\% ont un budget annuel inférieur à $5000 €$. Seules $1 / 5^{\mathrm{e}}$ ont une action nationale et $8 \%$ travaillent en relation avec l'international [ENQUÊTE REC, 1997]. La plupart s'attachent à des enjeux de défense de l'environnement local, quelques unes à la promotion de l'agriculture biologique. Les réseaux d'action hongrois en environnement sont donc désormais horizontaux et locaux ; ils semblent paradoxalement plus visibles au plan politique international qu'en Hongrie.

Du fait de cet ensemble de facteurs sociaux, la pression en faveur de l'environnement est donc moindre au sein de la sphère dirigeante. Le double manque, à la fois d'élus écologistes et d'un contrôle civique réellement militant fait que "ni les politiques, ni les décideurs économiques ne s'y intéressent plus » [MEZÖsI, id.]. Et c'est ce déficit de débat démocratique qui nous semble capital : la seule pression en matière d'environnement en Hongrie provient désormais de l'Union Européenne, c'est-à-dire d'une instance à la fois extra-nationale et institutionnelle. Si bien que la nature de l'action environnementale en Hongrie s'en trouve modifiée : elle tend à devenir centralisée, bureaucratique et partiale. Nous allons ainsi tenter de déceler certains de ces traits au sein même de ce qui semblerait participer de la rupture. 


\subsection{Des rémanences du socialisme dans l'actuelle politique environnementale}

La refonte du droit de l'environnement accompagne logiquement le besoin de rupture avec le socialisme et la volonté de convergence avec l'Union européenne : c'est un pré-requis des négociations d'adhésion exprimé dans le chapitre 22 sur l'environnement.

La loi n53 de 1996 et son décret d’application de 1997 constituent à cet égard une mise en conformité du droit hongrois avec l'acquis communautaire. Tous les aspects réglementaires de l'environnement y sont abordés en fixant précisément les compétences institutionnelles, les degrés de protection environnementale, les procédures d'agrément en urbanisme et les sanctions administratives. Un chapitre est notamment consacré à l'instauration de programmes agri-environnementaux et de plans de protection de la nature, tandis qu'un nouvel élan est donné aux services déconcentrés de l'environnement [BENCZE, 1996] : 12 inspections régionales (környezetvédelmi felügyelöségek), 13 directions des eaux (vízügyi igazgatóságok) et 10 directions des parcs nationaux (état 2003).

De même, l'État relance la politique des parcs nationaux en leur donnant de nouvelles fonctions de protection des paysages et de mise en valeur touristique locale. Certains sites retenus en 1997 sont très emblématiques, comme le Coude du Danube ou les forêts et coteaux viticoles des rives du lac Balaton.

Ceci n'empêche cependant pas certaines continuités organisationnelles de reprendre le dessus dès lors que l'action environnementale se replie de fait dans la sphère institutionnelle hongroise. Si l'on rappelle ici le concept de «dépendance du chemin », largement employé dans la réflexion sur la transformation systémique post-socialiste [STARK, BRUSZT, 1998], il existe bien des rémanences d'ordre structurel au sein des institutions issues du socialisme. Si les organismes changent de nom, si leurs fonctions sont remaniées par les nouveaux dirigeants, les personnels restent pour partie les mêmes et des routines comportementales se rétablissent aisément.

Ainsi, le Ministère de l'environnement s'était vu attribuer les compétences d'aménagement régional au sortir de 1990. Son travail s'ancrait résolument dans la perspective du développement durable avec un soutien aux initiatives endogènes et des perspectives de mise en valeur du patrimoine naturel et rural des régions hongroises. Pour des questions de politique intérieure et en signe d'allégeance envers le parti agrarien des Petits 
Propriétaires, l'aménagement régional a été transféré en 1998 au Ministère de l'agriculture. Ce changement n'est pas aussi neutre que l'on voudrait le dire [KAMPIS, 2003] : l'environnement se trouve ainsi coupé de sa perspective territoriale et opérationnelle tandis que le développement rural est désormais étroitement associé à la fonction agricole, signe d'une régression dans l'attention accordée à la diversité de l'espace rural.

De ce fait, les responsables locaux de l'environnement se heurtent désormais à un discours productiviste toujours vivace au sein du Ministère de l'agriculture [nos enquêtes, 2002-2003], lequel publiait encore en 1989 des articles discutant du potentiel cultivable de la Grande Plaine et des moyens d'intensifier l'irrigation [SZILÁRD, VÁRALLYAY, 1989]. C'est ce même ministère qui fait publier en 1999 le Programme agri-environnemental hongrois, base d'application des politiques européennes sur la multi-fonctionnalité rurale. Cet outil semble quelque peu caractérisé par une approche technique et sectorielle de l'environnement : il y a concertation mais peut-être moins participation. Or, à l'inverse, s'impose de manière croissante en Europe une approche à la fois globale et sociale des problèmes environnementaux.

Par ailleurs, l'organisme en charge de la planification de l'aménagement du territoire reste le VÁTI : certes privatisée, cette institution a élaboré tous les plans quinquennaux du socialisme. Il existe quelques personnalités de la mouvance écologiste hongroise dans ces institutions comme Károly JÁvOR, ingénieur au VÁTI, ancien militant du cercle du Danube et membre de Zöld Alternatíva : elles sont les premières à dénoncer l'inertie des institutions et le maintien de visions dépassées sur l'environnement et le développement rural dans les politiques d'aménagement hongroises [JÁVOR, 1997].

Autre choix significatif, les Directions des parcs nationaux se sont vues attribuer en 1996 des compétences administratives déconcentrées dans le contrôle de l'insertion paysagère du bâti. Cette tâche absorbe l'essentiel du temps des scientifiques des parcs tout en donnant un statut bureaucratique de censeur aux Directions. Quant aux parcs dernièrement créés, l'un semble avoir pour fonction principale de centraliser la gestion des réserves naturelles éclatées sur une région entière (Körös-Maros NP), un autre d'empêcher un projet frontalier d'industrialisation croate (Duna-Drava NP). Ces fonctions semblent peu compatibles avec les évolutions rencontrées ailleurs en Europe occidentale, où les parcs nationaux agissent en tant que catalyseurs du développement local. Les parcs s'avèrent même des partenaires utiles dans le montage de projets territoriaux en faveur du tourisme de nature et de la valorisation du patrimoine régional [DEPRAZ, 2003]. 
Dernière rémanence structurelle, certes moins spécifique à la scène hongroise que les points précédents, le territoire d'action des services déconcentrés de l'État ne correspond à aucun maillage administratif local ni au territoire des conseils régionaux en aménagement du territoire. Même au sein du ministère de l'environnement, les directions des eaux, de l'environnement et des parcs naturels ne sont pas en adéquation entre elles. C'est-à-dire que l'action environnementale se heurte, comme d'autres politiques d'intervention territoriale, à l'impossible décentralisation du pouvoir hongrois. La structuration verticale du pouvoir héritée du socialisme demeure en effet, jusqu'à ce jour, un obstacle à toute régionalisation.

Les objectifs affichés par les pouvoirs publics ont donc changé mais «la rupture est vite perceptible et perçue entre ceux qui proposent des nouveaux concepts, tels que le développement durable, et les techniciens-technocrates à l'œuvre, formés aux modèles plus classiques». Ces derniers "adoptent un vocabulaire plus environnementaliste que par le passé et évitent une remise en cause véritable » [KAMPIS, (332), id.].

La plus grande rémanence, cependant, peut paradoxalement provenir du manque d'initiative citoyenne que l'on a décrit plus haut comme un processus conjoncturel du postsocialisme [FODOR, 1998]. Peut-être cela est-il en fait, pour partie, également le signe d'une continuité avec la période socialiste - en particulier en milieu rural.

Si les sociétés locales avaient été à même de réagir aux questions environnementales et avaient fait montre d'une aptitude à la démocratie locale autour de 1988, il semble qu'elles soient revenues à une attitude plus passive caractéristique des temps socialistes : respect envers le pouvoir local, attente d'un rôle social des institutions publiques, faiblesse du débat politique. On peut attribuer cela à « une certaine désorientation après une longue période de politiques paternalistes »[KAMPIS, (334), id.]. Plus abruptement, n'est-ce pas là plutôt la traduction d'un maintien d'une tradition politique locale autoritaire, héritée du socialisme ? De fait, il s'avère que la plupart des dirigeants locaux, maires et conseillers municipaux, sont les mêmes qu'avant 1989. Nos entretiens auprès des élus locaux en Petite Coumanie ont montré que $59 \%$ des maires et $25 \%$ des élus sont issus, à des degrés divers, de l'appareil politique socialiste (anciens présidents du conseil, présidents de coopératives, cadres des fermes d'État, etc.). La présence d'une opposition municipale, politique ou associative, n'a permis un changement des responsables locaux que dans un cinquième des cas, en général dans les communes périurbaines au renouvellement social sensible. 
Dans les petites communes rurales, le mode de scrutin favorise la notabilité. Les élus portés au pouvoir par leur position sociale d'avant 1989 ont bénéficié de la prime au sortant et de l'absence d'alternative démocratique locale. De plus, pour se maintenir au pouvoir, ces élus ont généralement construit un nouveau discours identitaire teinté de valeurs paysannes largement artificielles. Dans ces représentations collectives, l'environnement est conçu comme un produit culturel local. Le territoire est donc un cadre de vie et une ressource, non une vitrine ou un espace à protéger de l'action anthropique. Il participe de l'image de stabilité et de conservatisme entretenue à dessein par les édiles locaux.

De ce fait, les politiques incitatives en matière de développement durable ou de protection de la nature sont généralement considérées comme exogènes : le poncif le plus fréquent de la part des élus est de dire que les écologistes et le pouvoir central «viennent protéger ce que nous avons su préserver seuls durant des siècles » [Entretiens, 2002-2003]. Ainsi le mouvement environnemental du tournant de 1989 aurait-il été progressivement transformé en un sentiment identitaire et localiste par des élites rurales installées, cherchant ainsi à préserver leur position dominante dans la tourmente de 1990. Cette attitude est largement contraire à l'impératif de concertation et de participation citoyenne des nouvelles politiques environnementales en Europe. Elle justifie pleinement l'action de terrain adoptée par la plupart des ONG environnementales hongroises mais laisse également à penser que leur tâche sera longue et ardue : leur réussite repose en effet sur l'effacement générationnel d'une rigidité sociale héritée, trace la plus durable du système socialiste.

\section{Conclusion}

En somme, l'action environnementale hongroise semble avoir connu deux temps forts depuis le début de la transformation post-socialiste. Le premier moment, marqué par l'effervescence démocratique du contrecoup des mouvements de la décennie quatre-vingts, met en place un paradigme dominant : celui de la rupture, du procès écologique du socialisme et de la mise en place d'une ligne politique intégrant l'environnement à tous les secteurs de l'action publique. Cependant, la participation citoyenne qui alimentait et soutenait ce grand changement s'effondre presque aussitôt.

Si bien qu'un deuxième temps s'esquisse depuis la seconde moitié de la décennie quatrevingt-dix, temps au cours duquel les politiques publiques en matière d'environnement poursuivent seules leur lancée en n'étant plus aiguillonnées que par l'impératif européen. De 
ce fait, elles prennent vite un tour technocratique et centralisateur, ce qui constitue un terreau favorable à l'affirmation de principes politiques hérités du socialisme : vision sectorielle de l'action territoriale, réticences à la délégation régionale voire locale et filtre bureaucratique croissant. Avec le temps, il semble également que le manque d'implication démocratique de la population hongroise dans les enjeux environnementaux ne soit pas qu'un reflux conjoncturel mais plutôt une rémanence de la structuration autoritaire des sociétés locales sous le socialisme.

Si la transformation post-socialiste a été rapide en matière économique, politique et administrative, elle s'avère donc bien plus longue lorsqu'il s'agit d'infléchir les habitudes sociales et les représentations collectives. L'affirmation d'un débat démocratique local est une base nécessaire aux politiques environnementales ; or c'est surtout cela qui fait défaut. Ce constat fondamental explique sans doute les échecs relatifs de l'écologie en Hongrie, en opposition sensible avec la tendance des politiques internationales.

\section{Références :}

BAtou J. (1992), «Révolution russe et écologie, 1917-1934 », in Vingtième siècle $\mathrm{n}^{\circ} 35$, Presses de la fondation nationale des Sciences Politiques, Paris, p. 16-28.

BENCZE L. (dir.) (1996), Act $n^{\circ}$ LIII of 1996 on Nature Conservation in hungary, OKTH / Ministère de l'environnement et du développement régional, Budapest, $48 \mathrm{p}$.

Bochniarz Z., Bolan R., KereKes S., Kindler J., VARgha J. VON WitZKe H. (1992), Environment and development in Hungary, a blueprint for transition, Budapest / Minneapolis, 88 p.

CSÁtARI B. (1995), «A special Regional environmental-social Conflict on the Great Hungarian Plain », in Potrykowska A., Clarke J.I. (dir.), «Population and Environment in industrial regions », Geographica Polonica n ${ }^{\circ}$ 64, Varsovie, p. 167-175.

ČĚ̌OvSKÝ J. (1988), Nature Conservation in the Socialist Coutries of East Europe, IUCN / Ministère de la culture, Prague, 116 p.

DEPRAZ, S. (2005), « Développement local et politiques de gestion des communes rurales en Hongrie centrale », in Bulletin de l'Association de Géographes Français vol. 82, Paris (à paraître).

DEPRAZ, S. (2003) - «Les parcs naturels en Hongrie, protection de la nature et développement local », in $L e$ Courrier des Pays de l'Est $\mathrm{n}^{\circ}$ 1035, La Documentation française, Paris, p. 71-79.

DEPRAZ, S., KerTÉSZ, Á. (2002) - «Évolution de la notion de protection de l'environnement en Hongrie, analyse géographique et sociale à travers l'exemple des parcs nationaux », in Annales de Géographie $\mathrm{n}^{\circ} 626$, S.E.D.E.S., Paris, p. 419-430.

EASTwOOD L. (1998), «From revolution to dissolution: Recent Transitions of the Eastern European Environmental Movement », in Carter F.W., Jordan P., Rey V., Central Europe after the Fall of Iron Curtain, Peter Lang Verlag coll. «Wiener Osteuropa Studien » vol. 4, Vienne, p. 175-186.

ENYEDI Gy., SzIRMAI V. (1998), «Environmental Movements and Civil Society in Hungary », in TICKLE A., WELSH I. (dir.), Environment and Society in Eastern Europe, Pearson Education, Harlow, p. 146-158.

ENYEDI Gy., HIRICHSEN D. (dir.) (1990), State of the Hungarian environment, Académie des Sciences / Ministère de l'environnement, Budapest, $143 \mathrm{p}$. 
ENYEDI Gy. (1974), «Planning for Purposeful Use of the Environment, a Hungarian Point of View », in VOLGYes I. (dir.), Environmental Deterioration in the SovietUnion and Eastern Europe, Praeger, New-York, p. 123-129.

FARAGÓ T., LAKOS A. (dir.) (1996), Approval and Implementation of International conventions on Environmental Protection and Nature Conservation in Hungary, Ministère de l'environnement et du développement régional, Budapest, $140 \mathrm{p}$.

FODOR I. (1998), «Impediments to sustainable development in the environmental Policy of East-Central Europe: the example of Hungary », in Kivell P., RoBerts P., WAlKer G.P. (dir.), Environment, Planning and Land Use, Ashgate, Aldershot / Brookfield, p. 147-156.

FODOR I., WAlKer G.P. (1994), Environmental Policy and Practice in Eastern and Western Europe, coll. «Environmental Studies » n¹1, Centre d'études régionales, Académie des Sciences, Pécs, 367 p.

FODOR I. (1991), « Changes in the State of the Environment in Hungary and their Impact on Human Health », in Specimina Geographica n², Centre d'études régionales, Académie des Sciences, Pécs, p. 116-126.

GARAMI L. (1993), Képes útikalauz, védett természeti értékeink (Guide illustré de nos monuments naturels protégés), Medicina könyvkiadó, Budapest, 272 p.

Guermond Y., Mathieu N. (dir.) (1991), «La question de l'environnement, naissance d'un débat en Pologne », in Strates, matériaux pour la recherche en sciences sociales $\mathrm{n}^{\circ} 6$, Paris, p. 3-100.

HARPER K. (1999), «Citizens or Consumers? Environmentalism and the Public Sphere in Postsocialist Hungary », in Radical History Review, n 74 , «Environmental Politics and Human Geographies », New-York, p. 96-111.

JÁVOR K. (1997), «Rural Areas and Issues of Rural Development in the Phase of Transition of Central-European Coutries - Hungarian Experience », in Papers of the ERSA Congress (European regional Sciences Association), Rome, CD-ROM, 14 p.

KAderJaK P., Powell J. (1997), Economics for Environmental Policy in Transition Economies : an Analysis of the Hungarian Experience, Edward Elgar publishing, coll. « New Horizons in environmental Economics », Cheltenham / Camberley / Northampton, 187 p.

KAMPIS B. (2003), «Les campagnes 'ateliers de développement' : expériences de développement rural durable en Hongrie », in ROMERO C. (dir.), Campagnes et société, fonctions et usages des campagnes françaises, Actes de colloque, CEDETE / Presses universitaires d'Orléans, Orléans, p. 328-336.

KALOTÁs Zs. (1996), Nemzeti parkjaink, jélen és jövö (les parcs nationaux hongrois, aujourd'hui et demain) Aqua Kiadó, Budapest, 116 p.

KERTÉSZ Á. et al. (1999), «Studies on the Impact of global Climate Change on some Environmental Factors in Hungary », in Időjárás, , vol. 103 n¹, Hungarian Meteorological Service, Budapest, p. 37-65.

KERTÉSZ Á. (1981), «Les zones de danger actuel ou virtuel de déflation en Hongrie », in KATONA S., KEREKES $\mathrm{S}$. (dir.), Recherches en environnement, cartographie et développement de l'environnement, $7^{\mathrm{e}}$ colloque francohongrois de géographie, Académie des Sciences, Budapest, p. 173-191.

KNABE B. (1989), « Glasnost für die Umwelt » (Glasnost pour l’environnement), in Osteuropa $\mathrm{n}^{\circ} 7$, p. 648-663.

MinistÈRE DE L'ENVIRONNEMENT HONGROIS (1996), Data on Hungary's environment, Budapest, 66 p.

Molnár B. (1998), «Protected geological objects in the Kiskunság National Park », in Geographica Pannonica $\mathrm{n}^{\circ} 2$, Faculté de mathématiques et de sciences naturelles, Université de Novi Sad, p. 5-11.

PÁJER J. (1992), Természetvédelem (protection de la nature), Egyetemi jegyzet, Sopron, 152 p.

PERSÁNYI M. (1993), « Red pollution, Green evolution, Revolution in Hungary », in JANKAR-WEBSTER B. (dir.), Environmental Action in Eastern Europe, Responses to Crisis, M.E. Sharpe, New-York, p. 134-157.

PERSÁNYI M. (1990), «Public participations and NGO : their role in Environmental Protection », in ENYEDI Gy, HINRICHSEN D. (dir.), The State of Hungarian Environment, Académie des Sciences, Budapest, 143 p.

PICKVANCE K. (1998), Democracy and Environmental Movements in Eastern Europe, Westview Press, Boulder (Colorado), $244 \mathrm{p}$.

RAKICS R., KovÁcs Á. (1996), Data on Human Environment, OKTH / Ministère de l'environnement et du développement régional, Budapest, $46 \mathrm{p}$.

R.E.C. (1997), Problems, Progress and Possibilities, a Needs Assessment of Environmental NGOs in central and Eastern Europe, Regional Environmental Center for Central and Eastern Europe, Aqua Kiadó, Szentendre, 96 p. 
SÁNDOR P. (1993), «New Directions in Environmental management in Hungary », in JANKAR-WEBSTER B. (dir.), Environmental Action in Eastern Europe, Responses to Crisis, M.E. Sharpe, New-York, p. 28-41.

Stark D., BruszT L. (1998) - Postsocialist Pathways ; transforming Politics and Property in East-Central Europe, Cambridge Studies in Comparative Politics, Cambridge University Press, Cambridge, 284 p.

SZABÓ L., KARÁCSONy J., SZÉKEly Zs. (1994), «Wind Erosion Problems in Hungary », in Agrokémia és Talajtan, vol. 43 n¹-2, Budapest, p. 109-112.

SzABÓ L. (dir.) (1985), Tudományos kutatások a Kiskunsági nemzeti parkban 1975-1984 (Recherches scientifiques dans le parc national du Kiskunság de 1975 à 1984), OKTH / KNP / HuNGEXPO, Budapest, 220 p.

SzILÁRD Gy., VÁRAllyay Gy. (1989), «Irrigation in Hungary » et « Soil Water Problems in Hungary » in Agrokémia és Talajtan, vol. 38 n³-4, Budapest, p. 577-595 et p. 685-690.

SZILASSY Z. (1990), « The Republic of Hungary », in ATKInSON R. (dir.), The environment in Eastern Europe, Gland, p. 34-41.

SZIRMAI V. (1997), «Protection of the Environment and the Position of Green Movements in Hungary », in LÁNG-PickVAnce K., MANning N., PiCKVAnCE C. (dir.), Environmental and Housing Movements ; Grassroots experiences in Hungary, Russia and Estonia, Aldershot, Avebury, p. 23-88.

SzUJKÓ-LACZA J. (1993), «Introduction to Flowering Plants in the Kiskunság National Park and the other Regions between the Danube and Tisza Rivers », in The Flora of the Kiskunság National Park, coll. « Natural History of the National Parks of Hungary », Muséum d'histoire Naturelle, Budapest, p. 43-61.

TARDY J. (dir.) (1996), Magyarországi települések védett természeti értékei (les monuments naturels protégés des localités hongroise), Mezőgazda Kiadó, Budapest, 664 p.

TóTH K. (dir.) (1996), Husz éves a Kiskunsági nemzeti park, tudományos kutatási eredmények (les 20 ans du parc national du Kiskunság, résultats des recherches scientifiques), KNP, Kecskemét, 234 p.

VÁRI A., TAMÁs P. (1993), Environment and Democratic Transition : Policy and Politics in Central and Eastern Europe, Kluwer Academic publishers, coll. « Technology, Risk and Society » vol. 7, Dordrecht, 296 p.

VINAVER K. (dir.) (1993), La crise de l'environnement à l'Est, pays en transition et expérience française d'une économie mixte, L'Harmattan coll. « Pays de l'Est », Paris, 190 p. 
Document 3 : chronologie thématique sur la protection de la nature et l'environnement en Hongrie

[synthèse d'après PAPP, KOVÁCS, 2001 ; LÁNG-PICKVANCE, 1997 ; REC, 1997 ; PERSÁNYI, 1993 \& 1990 ;

TARDY, 1996 ; PÁJER, 1992 ; ČEŘOVSKÝ, 1988]

Premières mesures de protection des ressources naturelles $\left(X V^{e} s .-X I X^{e} s.\right)$

1426 : règlement de Sigismond sur la gestion des forêts

1565 : loi sur l'organisation des forêts (régime des Habsbourg)

1669 : loi sur l'organisation des forêts (idem)

1729 : décret de Charles III sur la limitation de la chasse au gibier et aux oiseaux

1769 : décret de Marie-Thérèse sur l'entretien des forêts (éclaircissements, taillis)

1855 : décret réglementant la protection des forêts contre les feux

1879 : loi $\mathrm{n}^{\circ} 31$ sur la protection des forêts (charbonnages, feux, séjour, gibier, paysages)

1883 : loi $\mathrm{n}^{\circ} 20$ sur la chasse aux oiseaux et la pêche (périodes de fermeture)

1888 : décret d'application $\mathrm{n}^{\circ} 19$ listant des espèces protégées (poissons, oiseaux)

1893 : création du Centre ornithologique hongrois, aujourd'hui Institut d'étude des oiseaux

1894 : loi n ${ }^{\circ} 12$ limitant la pâture et l'accès des animaux aux terrains sableux

L'approche pittoresque et esthétisante sur la nature : 1900 - 1959

1900 : règlement listant des arbres remarquables, paysages naturels, espèces remarquables 1901 : décret d'application listant des espèces protégées en terrain sableux

1906 : décret portant création des « journée des oiseaux » et « journée des arbres »

1910 : première loi sur la « protection de la nature »; décret d'application avorté (1914)

1923 : loi n ${ }^{\circ} 18$ créant une autorité de protection de la nature au sein des services des forêts

1935 : loi n ${ }^{\circ} 4$ sur les forêts et la protection de la nature, sites naturels remarquables (Kaán)

1939 : création du Conseil national sur la protection de la nature (OTT), action à Debrecen

1945 : création de Bokor, un mouvement religieux, alternatif et environnementaliste

1952 : première réserve paysagère sur la presqu'île de Tihany

L'inflexion écologiste du kádárisme : 1960-1975

1961 : loi n 18 sur la protection de la nature, sanctions financières (théoriques) création de l'Office national de protection de la Nature $(\mathrm{OTH})$ loi $n^{\circ} 6$ sur la gestion des forêts et du gibier

1962 : décret $\mathrm{n}^{\circ} 23$ du 17 juin réglementant la protection des sites naturels et leur gestion

1970 : entrée de la Hongrie dans le programme MaB de l'Unesco, site du lac Fertő

1971 : décret $n^{\circ} 12$ du $1^{\text {er }}$ juin permettant la création de réserves et de parcs nationaux :

1972 : création du parc national de l'Hortobágy (ouest de Debrecen) sur $685 \mathrm{~km}^{2}$

1974 : fondation de la Société ornithologique Hongroise / Birdlife Hungary

1975 : création du parc national du Kiskunság (sud-est de Budapest) sur 350 km²
Emballement et politisation de l'action environnementale : 1976-1989

1976 : lancement du programme de protection de la nature sur 15 ans, objectifs chiffrés création du parc national de Bükk sur $388 \mathrm{~km}^{2}$, entre Miskolc et Budapest

1977 : décret n²3 élargissant le rôle de l'OTH, de l’OTT et créant des directions régionales accord hungaro-tchécoslovaque pour la construction d'un barrage sur le Danube

1980 : création de l'Institut pour la protection de l' environnement (KVI), agence de mesure

1981 : création civile de l'Interdiszciplináris Tudományos Diákkör ; conférences, ateliers mouvement civil contre la pollution de l'eau potable par déchets industriels à Vác mouvement civil contre un projet d'incinérateur des ordures ménagères à Zsámbék

1982 : décret $\mathrm{n}^{\circ} 4$ et application $\mathrm{n}^{\circ} 8$ (du 15 mars) instaurant un droit de l'environnement

1983 : création du Club de protection de la nature de l'Université ELTE à Budapest mise en ouvre du programme d'assainissement du lac Balaton

1984 : fondation du Cercle du Danube en réaction contre le projet de barrage sur le fleuve

1985 : le Cercle du Danube reçoit le Right Livelihood, prix international en écologie création du parc national d'Aggtelek, au nord de Miskolc $\left(198 \mathrm{~km}^{2}\right)$

1986 : lancement de Greenway, un réseau européen étudiant de protection de la nature

1987 : création du Ministère de l'environnement et de l'eau par fusion OKTH et office eaux mouvement civil contre une pollution au plomb issue de la métallurgie à Nagytetény

1988 : fusion des 7 sous-directions régionales de l'environnement et de l'eau

loi autorisant la création d'associations civiles indépendantes du parti communiste mouvement civil contre un projet de dépôt nucléaire à Ófalu et Püspökszilágy

contre des décharges à Monorierdő, Apajpuszta, Aszod, Kuncsorba et Kápolnásnyék contre une usine de batteries au plomb à Gyongyösoroszi

contre un projet de mine de bauxite à Hévíz, à proximité du lac Balaton

1989 : tables rondes, chute du parti social-ouvrier hongrois, élections libres

Depuis 1990 : désaffection civile et institutionnalisation de l'environnement

1990 : attribution de l'aménagement du territoire au ministère de l'environnement 1991 : création d'un parc bi-national sur le lac Fertő (parc du Fertö-Hansag, 198 km²)

1993 : fondation du parti Zöld Alternatíva, lutte contre les choix gouvernementaux, échec

1996 : loi n53 sur la protection de l'environnement / sur la protection de la nature

1997 : création de 4 parcs nationaux aux fonctions décentralisées de contrôle des paysages

1998 : transfert de l'aménagement du ministère de l'environnement vers l'agriculture

1999 : Publication du Programme agri-environnemental national

2002 : création du $10^{\mathrm{e}}$ parc national hongrois dans l'Örség

2004 : la Hongrie compte 9\% de son territoire placé sous un statut de protection 